\title{
Ab initio four-band Wannier tight-binding model for generic twisted graphene systems
}

\author{
Jin Cao, ${ }^{1}$ Maoyuan Wang, ${ }^{1,2}$ Cheng-Cheng Liu, ${ }^{1, *}$ and Yugui Yao ${ }^{1, \dagger}$ \\ ${ }^{1}$ Key laboratory of advanced optoelectronic quantum architecture and measurement (MOE), \\ School of Physics, Beijing Institute of Technology, Beijing 100081, China \\ ${ }^{2}$ International Center for Quantum Materials, School of Physics, Peking University, Beijing 100871, China
}

(Dated: December 7, 2020)

\begin{abstract}
The newly realized twisted graphene systems such as twisted bilayer graphene (TBG), twisted double bilayer graphene (TDBG), and twisted trilayer graphene (TTG) have attracted widespread theoretical attention. Therefore, a simple and accurate model of the systems is of vital importance for the further study. Here, we construct the symmetry-adapted localized Wannier functions and the corresponding $a b$ initio minimal two-valley four-band effective tight-binding models for generic twisted graphene systems with small twist angle. Such two-valley model evades the Wannier obstruction caused by the fragile topology in one-valley model. The real space valley operator is introduced to explicitly describe the valley $U_{v}(1)$ symmetry. Each symmetry-adapted Wannier orbital shows a peculiar three-peak form with its maximum at AA spots and its center at AB or BA spots. An extended Hubbard model is also given and the related parameters are presented explicitly. We provide an approach to systematically build the Wannier tight-binding model for generic twisted graphene systems. Our model provides a firm basis for further study of the many-body effects in these systems.
\end{abstract}

Introduction. - The recent discovery of correlated insulating states and possibly unconventional superconductivity in magic-angle twisted bilayer graphene (TBG) $[1$, 2] has triggered broad interest in TBG systems[1-40]. Immediately after the magic-angle TBG, twisted double bilayer graphene (TDBG)[41-49] and twisted multilayer graphene (TMG)[50-55] as well as other twisted twodimensional materials[56-61] have been fabricated and investigated, forming a new research field - twistronics. Generic TBG systems are usually described by effective continuum model[62-64], tight-binding (TB) model[52, 59, 65-69] and density functional theory (DFT) [58, 7074]. However, these models need lots of basis to model the single-particle band structure. Even for the continuum model, hundreds of basis are needed, and the other two need tens of thousands and even more, which has seriously hindered the study of the novel many-body quantum states in twisted graphene systems.

For TBG with small twist angle, two kinds of effective Wannier TB models with several orbitals were proposed[18-23]. One is building the effective Wannier TB model for one valley, which we refer to $1 \mathrm{~V}$ TB model. Due to the so-called fragile topology in the low-energy bands, more deliberated-selected extra trivial orbitals should be added to form a wannierizable group[18, 22, 24, 25]. Obviously, this method cannot be directly promoted to generic twisted graphene systems. The other is considering two valleys together named $2 \mathrm{~V}$ TB model[19-21]. The low-energy flat bands for 2V-TB model in TBG represent a trivial band topology[22, 25]. The $1 \mathrm{~V}$-TB model separates two valleys, which explicitly preserves the $U_{v}(1)$ valley symmetry, while such symmetry might be lost in $2 \mathrm{~V}-\mathrm{TB}$ model.

Here, we attempt to provide a numerical method to systematically construct $a b$ initio minimal four-band
Wannier TB models for arbitrary stacked graphene systems with a small twist angle. First, from ab initio calculations, the band structures of untwisted multilayer graphene subsystems are obtained. Our ab initio calculations naturally take into account the trigonal warping around the graphene valleys induced by interlayer coupling[75]. It was often ignored in previous studies and should be included since the energy scales for the trigonal warping and the flat bands after twist are comparable. We numerically explicitly demonstrate the chiral decomposition rule for TMG, and identify the low energy bands and the distribution in real space. Given this knowledge, the Wannier functions (WFs) are constructed by combining the microscopic $p_{z}$ orbitals with an envelope function. The TB model for $\operatorname{TBG}(1+1), \operatorname{TDBG}(2+2)$, twisted trilayer graphene $(\mathrm{TTG})(1+2)$ and TMG $(4+4)$ are constructed as examples. Based on the WFs for these systems with small twist angle, we explicitly present the real space Hamiltonian and the valley operator, which together encode the whole low-energy physics and the symmetry, especially the $U_{v}(1)$ valley symmetry. Finally, the electron-electron interactions between the localized WFs is discussed and the extended Hubbard model is given.

Chiral decomposition of few-layer and twisted multilayer graphene. - Stacking single-layer graphene (SLG) along $\hat{\mathbf{z}}$ direction forms a few-layer graphene (FLG) system. The energetically favorable stacking order is generated by intralayer translations along $\left(\mathbf{a}_{1}+\mathbf{a}_{2}\right) / 3$ with an additional interlayer $d_{0} \hat{z}$ resulting in three nonequivalent layers labeled by $\mathrm{A}, \mathrm{B}$ and $\mathrm{C}$ respectively[76], as shown in Fig.1(a). Here $d_{0}$ represents the layer distance of FLG. One stacking order is referenced as chirally stacking order if all of the intralayer translations are the same. Clearly a general stacking sequence can be decomposed into several subsets of chirally stack- 

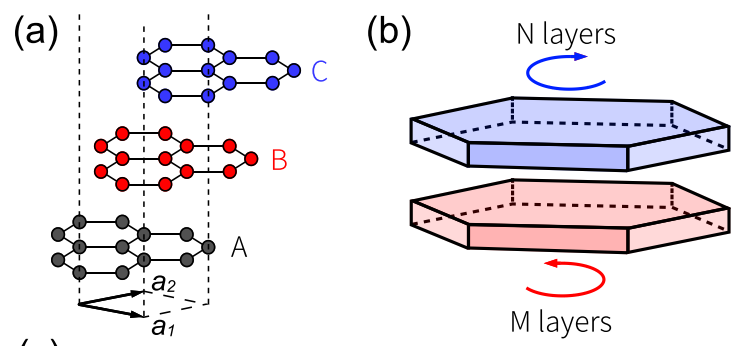

(c)

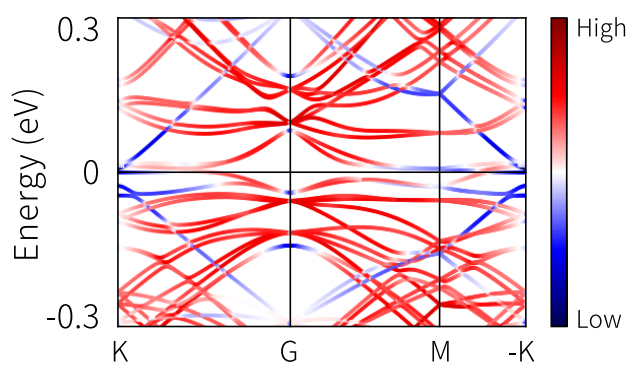

FIG. 1. (a) Stacked nonequivalent layers in few-layer graphene labeled as A, B and C respectively. (b) Generic twisted multilayer graphene system with $\mathrm{M}$ and $\mathrm{N}$ layers arbitrary stacked graphene on the bottom and top respectively denoted as $\mathrm{TMG}_{\mathrm{M}}^{\mathrm{N}}$. (c) Chiral decomposition for $\mathrm{TMG}_{\mathrm{M}}^{\mathrm{N}}$, where $\mathrm{M}=\{\mathrm{ABCA}\}$ and $\mathrm{N}=\{\mathrm{ABCA}\}\{\mathrm{C}\}$. The band structures are projected onto two nearest active chiral subsets, i.e., the $\{\mathrm{ABCA}\}$ chiral subset on the bottom and the $\{\mathrm{ABCA}\}$ chiral subset on the top.

ing order, the so-called chiral decomposition in FLG[77]: The low energy states of the N-layer stacked graphene can be well described by direct sum of $N_{\mathrm{D}}$ subspaces, $H_{N}^{\mathrm{eff}} \approx H_{J_{1}} \oplus \cdots \oplus H_{J_{N_{\mathrm{D}}}}$, where each of $H_{J_{i}}$ is a pseudospin doublet with $k^{J_{i}}$ leading order dispersion induced from the $i$ th chiral subset in N-layer stacked graphene with the sum rule $\sum_{i=1}^{N_{\mathrm{D}}} J_{i}=N$. Furthermore, these low energy states are localized at the boundary of the chirally stacking subsets. For example, a chirally stacking $J_{i}$-layer subset has $J_{i}-1$ pairs of dimmer and two unpaired sites left. The two unpaired sites contribute two zero modes, which are responsible for the low energy subspace, and the rest parts are pushed into high energy by strong direct interlayer coupling. The previous studies usually considered the ideal case, where are only the nearest interlayer hopping parameters taken into account, and ignored the trigonal warping and particle-hole $(\mathrm{PH})$ asymmetry in realistic case. We would like to point out that the effects should be included because of the comparable energy scale with that of the low-energy flat bands. In this work, the electronic structure for FLG are obtained from $a b$ initio calculations, where the trigonal warping and $\mathrm{PH}$ asymmetry are automatically included (see Appendix.A for details). The ab initio results show a well preserved chiral decomposition rule as presented in Appendix.B.

Considering a general TMG system, as presented in
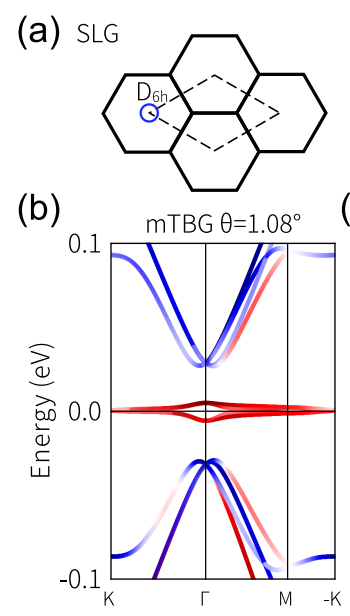

(c)
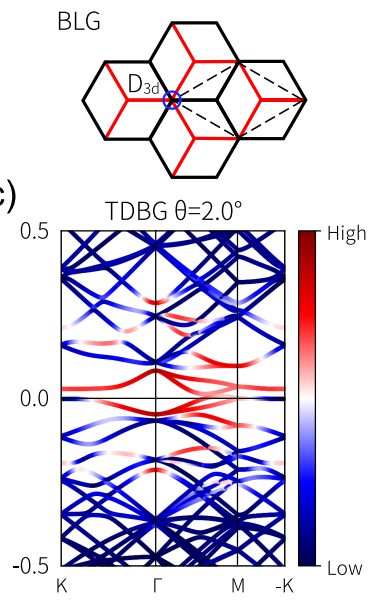

FIG. 2. (a) Maximum site symmetry for SLG and BLG as circled in blue. The black and red solid lines stand for layers. (b) and (c) Band projections onto the initial WFs for TBG and TDBG $\left(\mathrm{TMG}_{\{\mathrm{AB}\}}^{\{\mathrm{AB}\}}\right)$. The flat bands can be well described by the initial WFs by means of the large overlap. And it is guaranteed since these WFs notably overlap with the zero modes, which are responsible for the low energy states.

Fig.1(b), it has N-layer graphene on the top and M-layer graphene on the bottom with small twist angles $\pm \theta / 2$ respectively. In the absence of twisted interlayer coupling, this system is described by several groups of pseudospin doublets with $k^{J_{i}}$ leading order dispersion. With the twisted interlayer coupling turned on, in fact two active chiral subsets are responsible for the flat bands: the bottom chiral subset of the upper $\mathrm{N}$ layers and the top chiral subset of the lower M layers. From the continuum model, we numerically calculated the orbital characters for TMG system. We choose $\mathrm{TMG}_{\{\mathrm{ABCA}\}}^{\{\mathrm{ABCAC}\}}$ with a relative twist angle $2.646^{\circ}$ as an example. It can be decomposed into three chiral subsets $\{\mathrm{ABCA}\},\{\mathrm{ABCA}\}$ and $\{\mathrm{C}\}$ in quartic and linear dispersion. As presented in Fig.1(c), the former two active twisted chiral subsets strongly renormalize into the flat bands, and the left one preserves well the linear dispersion. In brief, the low-energy states in TMG can be well described by two decoupled parts: the renormalized flat bands and the left pseudospin doublet.

Four-band Wannier tight-binding model for generic TMG. - We start by discussing the symmetry of TMG. The space groups of all FLG are symmorphic, i.e., apart from the lattice translations, all of the symmetric operations leave one site fixed [see Fig.2(a)]. When twisted, the symmetry group depends on the twist center. To get the maximal symmetric structure in real space, one should take the twist center at that of the maximal site symmetry. The symmetry of the band structure is not sensitive to this atomic level selection since the moire pattern is much larger than the atomic length scale. The nonzero twist angle removes the inversion symmetry. Based on 
TABLE. I. Symmetry of the twisted multilayer graphenes.

\begin{tabular}{ccc}
\hline \hline Stacking order & Point Group & Generators \\
\hline $\mathrm{TBG}$ & $D_{6}$ & $\mathcal{C}_{6 z}, \mathcal{C}_{2 x}$ \\
$\mathrm{TMG}_{\{\mathrm{AB}\}}^{\{\mathrm{AB}\}}(\mathrm{TDBG})$ & $D_{3}$ & $\mathcal{C}_{3 z}, \mathcal{C}_{2 x}$ \\
$\mathrm{TMG}_{\{\mathrm{AB}\}}^{\{\mathrm{BA}\}}(\mathrm{TDBG})$ & $D_{3}$ & $\mathcal{C}_{3 z}, \mathcal{C}_{2 x}$ \\
generic $\mathrm{TMG}_{\mathrm{N}}^{\mathrm{M}}$ & $C_{3}$ & $\mathcal{C}_{3 z}$ \\
\hline \hline
\end{tabular}

this knowledge, the maximal symmetry groups for the twisted graphene systems are given in Table.I. The $\mathcal{C}_{3 z}$ symmetry is in general preserved.

In the absence of twisted interlayer coupling, the low energy Bloch states in TMG can be viewed as folding the band structure of FLG, which are induced from the microscopic $p_{z}$ orbitals of FLG. Also, these low energy Bloch states come from the graphene valleys thus taking a high frequency factor $e^{i \mathbf{K}_{\xi}^{\mathrm{FLG}} \cdot \mathbf{r}}$, where $\mathbf{K}_{\xi}^{\mathrm{FLG}}$ is the valley of FLG. It suggests that the WFs can be explicitly written in the form

$$
\left|g_{n}\right\rangle=\frac{1}{2} \sum_{\xi ; \tau, d, \mathbf{R}} e^{i \mathbf{K}_{\xi}^{\mathrm{FLG}} \cdot \mathbf{r}} f_{n}^{(\xi ; \tau, d)}(\mathbf{r})|\tau, d, \mathbf{R}+\mathbf{d}\rangle .
$$

Here $|\tau, d, \mathbf{R}+\mathbf{d}\rangle$ is the microscopic $p_{z}$ orbitals of FLG and the sum runs over valley index $\xi$, sublattice $\tau$, layer index $d$ and graphene lattice $\mathbf{R}$. $n$ is the index of the WFs, and $\tau=\tau_{\alpha}, \tau_{\beta}$ denotes the position of the sublattice. $f_{n}^{(\xi ; \tau, d)}$ is the smooth envelope function in moiré length scale. Since we consider a $2 \mathrm{~V}$-TB model, the entire system preserves the time reversal symmetry. It is possible to choose a group of real-valued WFs with constraint $f_{n}^{\left(\xi_{+} ; \tau, d\right)}=f_{n}^{\left(\xi_{-} ; \tau, d\right)} \equiv f_{n}^{(\tau, d)}$. It should be pointed out that different from the 1V-TB model, in which the valley degrees of freedom are promoted as orbitals, in the $2 \mathrm{~V}$-TB model, the valley degrees of freedom are denoted as orbital components. Also, our choice of WFs equally mixes two valleys. This mixture cannot be removed due to the non-trivial topology of the flat bands from a single valley. Despite of this choice, it is possible to preserve well the valley $U_{v}(1)$ symmetry as we will discuss later.

For generic TMG, we choose the envelope function for the initial WFs as $f_{1}^{\left(\tau_{\alpha},-1\right)}(\mathbf{r})=G\left(\mathbf{r}-\mathbf{r}_{1}^{\text {hex }}\right)$, $f_{2}^{\left(\tau_{\beta},-1\right)}(\mathbf{r})=G\left(\mathbf{r}-\mathbf{r}_{2}^{\text {hex }}\right), f_{3}^{\left(\tau_{\beta}, 1\right)}(\mathbf{r})=-G\left(\mathbf{r}-\mathbf{r}_{1}^{\text {hex }}\right)$ and $f_{4}^{\left(\tau_{\alpha}, 1\right)}(\mathbf{r})=-G\left(\mathbf{r}-\mathbf{r}_{2}^{\text {hex }}\right)$. Here $G\left(\mathbf{r}-\mathbf{r}_{i}^{\text {hex }}\right)$ is the Gaussian function localized at the hexagonal site with moiré scale spreading. The initial choice is based on the following considerations. Firstly, it reflects the realistic orbitals character since these chosen WFs have notable overlap with the relevant flat bands, as explicitly shown in Fig.2. Secondly, the initial choice respects the corresponding symmetry: (i) Each $g_{n}$ itself has $\mathcal{C}_{3 z}$ symmetry due to the site symmetry at hexagonal site. The $\mathcal{C}_{3 z}$ symmetry is preserved for all TMG systems. (ii) In each
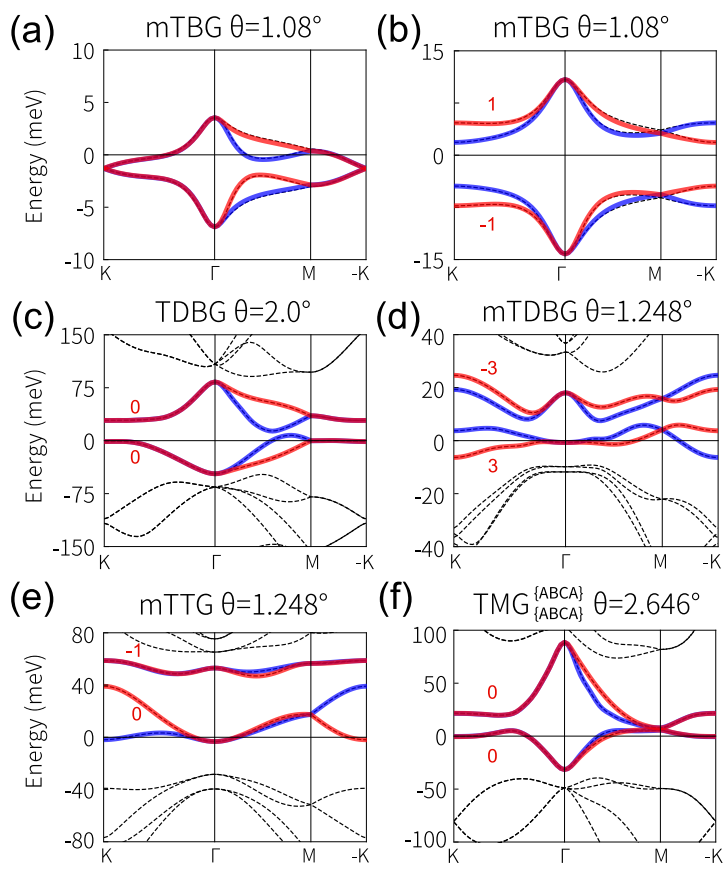

FIG. 3. Band structures interpolated from Wannier tightbinding model (in red and blue lines) for (a) TBG, (b) TBG on h-BN substrate, (c) TDBG, (d) magic angle TDBG, (e) magic angle TTG and (f) $\mathrm{TMG}_{\{\mathrm{ABCA}\}}^{\{\mathrm{ABCA}\}}$ respectively, with the effects of atomic relaxations taken in to account. As a comparison, the band structures by combining the effective continuum model and $a b$ initio calculation are given in black dash lines. Our Wannier tight-binding band structures are obtained by simultaneously diagonalizing the Hamiltonian and the valley operator, and thus can be labeled with a well-defined valley eigenvalues $\pm 1\left(\mathbf{K}, \mathbf{K}^{\prime}\right)$ highlighted in red and blue lines.

graphene layer, $\mathcal{C}_{2 z}$ exchanges the sublattice degrees of freedom, thus exchanges $g_{1}, g_{3}$ with $g_{2}, g_{4}$ respectively. The $\mathcal{C}_{2 z}$ symmetry is only preserved in TBG. (iii) $\mathcal{C}_{2 x}$ exchanges $g_{1}, g_{2}$ with $g_{3}, g_{4}$ respectively. The $\mathcal{C}_{2 x}$ symmetry is preserved in TBG and TDBG.

Given these envelope functions, one can construct the WFs and the related TB model following the methodology developed by D. Vanderbilt et al. [78, 79] (see Appendix.D for details). The Wannier TB models and the WFs for $\mathrm{TMG}_{\mathrm{N}}^{\mathrm{M}}$ are automatically produced by our home-made code. With the TB model of the few-layer graphene from $a b$ initio calculations (see Appendix.A), one can readily obtain the four-band Wannier TB model for generic TMG with small twist angle. Here we build TB models for several prototypical TMG systems for examples, i.e., mTBG with twist angle $1.08^{\circ}$, mTBG with magic angle $1.08^{\circ}$ and h-BN substrate $\Delta_{\mathrm{BN}}^{d=-1}=$ $30 \mathrm{meV}$, TDBG with twist angle $2.0^{\circ}$, mTDBG with magic angle $1.248^{\circ}$ and displacements field tuned on $\left(U_{\mathrm{D}}=10 \mathrm{meV}\right), \operatorname{mTTG}\left(\mathrm{TMG}_{\{\mathrm{A}\}}^{\{\mathrm{AB}\}}\right)$ with magic angle $1.248^{\circ}\left(U_{\mathrm{D}}=80 \mathrm{meV}, \Delta_{\mathrm{BN}}^{d=-1}=80 \mathrm{meV}\right.$ and $\Delta_{\mathrm{BN}}^{d=1}=$ 
(a)
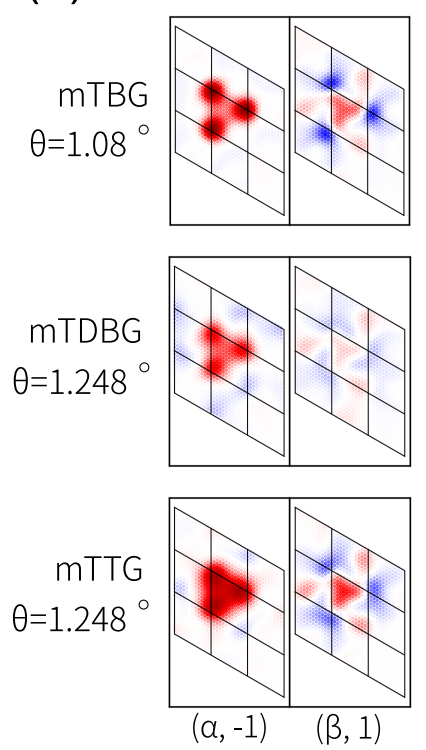

WF2
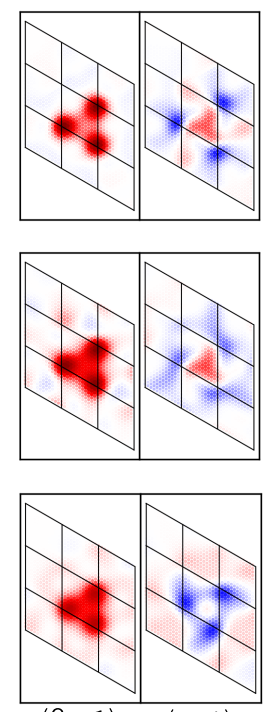

$(\beta,-1) \quad(\alpha, 1)$
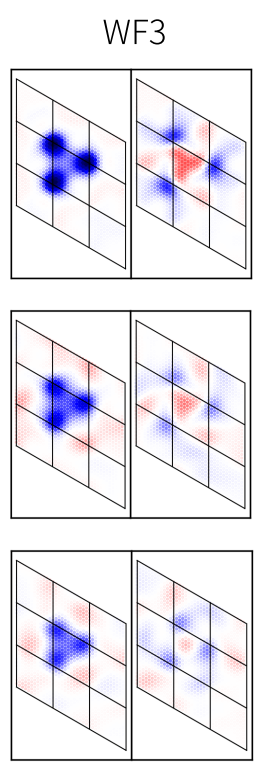

$(\beta, 1) \quad(\alpha,-1)$
WF4
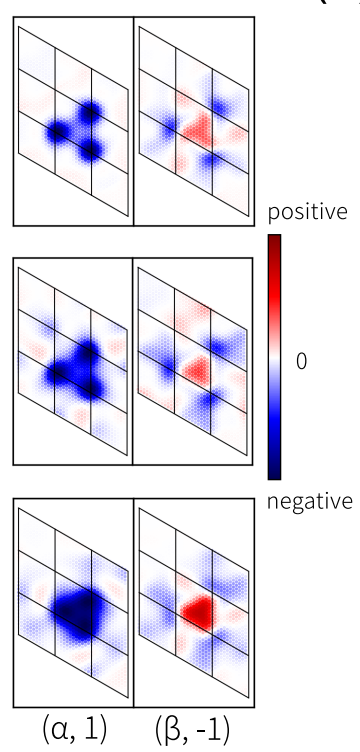

(b)

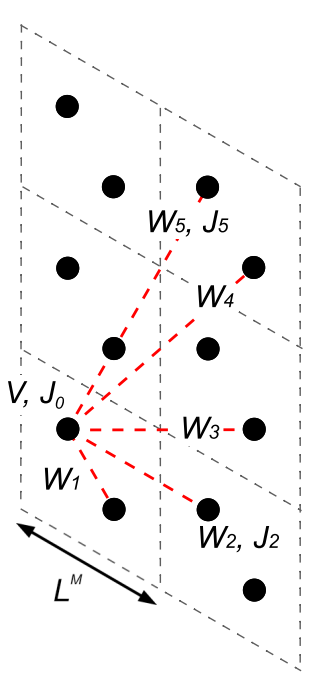

FIG. 4. (a) Plot of the envelope part $f_{n}^{(\xi ; \tau, d)}(\mathbf{r})$ of $C_{3 z}$-symmetry-adapted localized WFs for mTBG with magic angle $\theta=1.08^{\circ}$, mTDBG with magic angle $\theta=1.248^{\circ}$ and mTTG with magic angle $\theta=1.248^{\circ}$ respectively. Only $\mathbf{K}$ valley case is presented, the another one is the same. The $3 \times 3$ moiré cell is shown in black lines. (b) Nonzero Coulomb interactions between these WFs. The extended Hubbard interactions are taken into account due to the extended features of the WFs. For exchange interactions, the nonzero terms presented here are $J_{0}, J_{2}$ and $J_{5}$. The other terms, i.e., $J_{1}, J_{3}$ and $J_{4}$ are interactions between the two WFs, which are located at different layers or sublattices resulting in zero exchange interactions. Notice that WFs have three peaks at the moiré triangular lattice sites (a) but are centered at the dual honeycomb lattice sites (b).

$\Delta_{\mathrm{BN}}^{d=2}=-20 \mathrm{meV}$ ), and $\mathrm{TMG}_{\{\mathrm{ABCA}\}}^{\{\mathrm{ABCA}\}}$ with twist angle $2.646^{\circ}$. They band structures are shown in Fig.3, which fit well with the effective continuum model. The $\mathcal{C}_{3 z}$ symmetry is enforced in building symmetry-adapted WFs since it is preserved in all TMG system, and also reflected in the hopping parameters between WFs, as plotted in Figs.S.4-S.6. The WFs show a peculiar three-peak form, as shown in Fig.4(a). Beside the one component of each Wannier orbital we set up initially, there emerges another component located at same hexagonal site but in different layer and sublattice. It should be pointed out this is different from the previous studies[20, 21], where each component is nonzero for all of the WFs. Our Wannier TB model well describes the Berry curvature distributions and Chern numbers of generic TMG, e.g., TBG, TDBG and so on, with zero valley Chern number, i.e., the sum of Chern number of the two flat bands for single valley is zero. The Berry curvature distributions and Chern numbers for both valleys are given in Appendix.E. Although the topological description of TMG with nonzero valley Chern number is beyond our current Wannier TB model, the model fits the four flat bands very well for generic TMG with or without nonzero valley Chern number. As an example of TMG with nonzero valley Chern number, mTTG has zero Chern number for the valence band and nonzero one for the conduction band, which suggests one may include higher energy bands rather than the four flat bands to characterize the topological aspect.

An important feature for twisted graphene systems with small twist angle is the well preserved valley $U_{v}(1)$ symmetry due to the negligible intervalley coupling. The $U_{v}(1)$ symmetry is explicitly present in continuum model. However, such symmetry does not seem to exist in our two-valley Wannier TB model. In fact, our method indeed manifests well the $U_{v}(1)$ symmetry since the initial Bloch sum is unitarily transformed from the original flat bands obtained by the continuum model [see Eq.(D6)]. To reveal the valley degrees of freedom in our $2 \mathrm{~V}$-TB model, we define the valley operator for the continuum model, and the valley operator for our Wannier TB model can be interpolated from the exactly same procedure as that of Hamiltonian as illustrated in Appendix.D. The eigenvalues of interpolated valley operator are stabilized at \pm 1 as shown in Fig.S.3. By simultaneously diagonalizing the Hamiltonian and the valley operator, the bands and eigenstates in our Wannier TB model can be labeled with valley eigenvalues, and thus the $U_{v}(1)$ symmetry is preserved. The bands with different valley eigenvalues $\pm 1\left(\mathbf{K}, \mathbf{K}^{\prime}\right)$ are colored in blue and red in Fig.3.

The extended Hubbard model for generic TMG.- Finally, we discuss the electron-electron interactions between the constructed localized WFs. Because each Wannier orbital has two nonzero components out of the total 
TABLE. II. Coulomb interaction between WFs in unit of $\frac{e^{2}}{4 \pi \epsilon L^{\mathrm{M}}}$, which are integrated in a $5 \times 5$ moiré lattice. Taking $\epsilon_{r}=5$ for a BN substrate, the unit for mTBG $\left(\theta=1.08^{\circ}\right)$ is $22 \mathrm{meV}$ and are $26 \mathrm{meV}$ for $\mathrm{mTDBG}\left(\theta=1.248^{\circ}\right)$ and $\mathrm{mTTG}$ $\left(\theta=1.248^{\circ}\right)$. The $\mu$ and $\bar{\mu}$ represent for different orbitals.

\begin{tabular}{cccc}
\hline \hline & $\mathrm{mTBG}\left(1.08^{\circ}\right)$ & $\mathrm{mTDBG}\left(1.248^{\circ}\right)$ & $\mathrm{mTTG}\left(1.248^{\circ}\right)$ \\
\hline$U$ & 2.210 & 1.991 & 2.330 \\
$V$ & 2.208 & 2.011 & 2.150 \\
$W_{1}$ & 1.742 & 1.636 & 1.689 \\
$W_{2}$ & 1.238 & 1.151 & 1.149 \\
$W_{3}$ & 1.160 & 1.053 & 1.029 \\
$W_{4}$ & 0.700 & 0.761 & 0.717 \\
$W_{5}$ & 0.623 & 0.653 & 0.615 \\
$J_{0}^{\mu \bar{\mu}}$ & 0.000 & 0.016 & 0.253 \\
$J_{2}^{\mu \mu}$ & 0.192 & 0.117 & 0.070 \\
$J_{2}^{\mu \bar{\mu}}$ & 0.044 & 0.036 & 0.049 \\
$J_{5}^{\mu \mu}$ & 0.002 & 0.017 & 0.002 \\
$J_{5}^{\mu \bar{\mu}}$ & 0.003 & 0.006 & 0.006 \\
\hline \hline
\end{tabular}

four components and shows the peculiar three-peak form, the interaction Hamiltonian takes the following form

$$
\begin{aligned}
H_{i n t}= & U \sum_{i \mu} n_{i \mu \uparrow} n_{i \mu \downarrow}+V \sum_{i \mu>\nu} n_{i \mu} n_{i \nu} \\
& +\frac{1}{2} \sum_{\alpha} W_{\alpha} \sum_{\langle i j\rangle_{\alpha}, \mu \nu} n_{i \mu} n_{j \nu} \\
& +\sum_{i \mu>\nu, \sigma \sigma^{\prime}} J_{0} a_{i \mu \sigma}^{\dagger} a_{i \nu \sigma} a_{i \nu \sigma^{\prime}}^{\dagger} a_{i \mu \sigma^{\prime}} \\
& +\sum_{i \mu \neq \nu} J_{0} a_{i \mu \uparrow}^{\dagger} a_{i \nu \uparrow} a_{i \mu \downarrow}^{\dagger} a_{i \nu \downarrow} \\
& +\frac{1}{2} \sum_{\langle i j\rangle_{2} \mu \nu, \sigma \sigma^{\prime}} J_{2}^{\mu \nu} a_{i \mu \sigma}^{\dagger} a_{j \nu \sigma} a_{j \nu \sigma^{\prime}}^{\dagger} a_{i \mu \sigma^{\prime}} \\
& +\sum_{\langle i j\rangle_{2} \mu \nu} J_{2}^{\mu \nu} a_{i \mu \uparrow}^{\dagger} a_{j \nu \uparrow} a_{i \mu \downarrow}^{\dagger} a_{j \nu \downarrow}
\end{aligned}
$$

where $a_{i \mu \sigma}^{\dagger}$ creates a WFs at $i$ site with spin index $\sigma$. $\mu=g_{1}, g_{3}$ or $g_{2}, g_{4}$ depending on the location of the Wannier center. The numerical integration values of the parameters in the interaction Hamiltonian for mTBG, mTDBG and mTTG are summarized in Table.II. The extended Hubbard interactions are represented by the $W_{\alpha}(\alpha=1 \sim 5)$ For the first three terms, the quantitively difference is mainly determined by the distance between the localized WFs. The two WFs, which are in the same shape but located in different layers or sublattice, have little effect on the direct interactions. For Hund's exchange and pair-hopping terms, the nonzero terms are shown in Fig.4(b). It is nonzero only for the WFs located at the same hexagonal site up to the lattice translations. The reason is that, for instance the nonzero components of $g_{1}$ and $g_{2}$ are located at different layers or sublattices resulting in a zero exchange interaction.
Conclusion and discussion.- We present an approach to construct $\mathcal{C}_{3 z \text {-symmetry-adapted localized }}$ WFs and the corresponding ab initio minimal fourband effective tight-binding models for generic twisted graphene systems with small twist angle, such as experimentally realized TBG, TDBG, and TTG, as well as other $\mathrm{TMG}_{\mathrm{M}}^{\mathrm{N}}$ systems experimentally to be realized. Each symmetry-adapted Wannier orbital shows a peculiar three-peak form with two nonzero components out of the total four components. An extended Hubbard model is also obtained and the related parameters are calculated explicitly.

Our starting point is the $a b$ initio band structures of untwisted multilayer graphene subsystems, which naturally includes the trigonal warping effect, which was often ignored in previous study and should be taken into account since the energy scales for the trigonal warping and the flat band after twist are comparable. Then we use the continuum model to address the twisted cases with the lattice relaxation effect taken into account. For a singlevalley model, constructing two-band Wannier model will have an obstruction due to the fragile topology in TBG systems, but for a two-valley four-band model, it is wannierable. Moreover, the valley $U_{v}(1)$ symmetry can still be retrieved in our two-valley four-band model. We can use the eigenvalue of the valley operator $\pm 1\left(\mathbf{K}, \mathbf{K}^{\prime}\right)$ to mark the energy band by constructing the valley operator explicitly, and then diagonalizing it with the Hamiltonian simultaneously. The effect of external electric displacement field and sublattice symmetry breaking can be readily incorporated in our model. Our ab initio minimal four-band effective Wannier tight-binding models together with the extended interactions is of importance for a wide range of applications in the efficient study of the many-body effects in the TMG systems.

C.-C. L thanks F. Yang, L.-D. Zhang for earlier related collaborations on TBG, and J. Liu for fruitful discussions. This work was supported by the NSF of China (Grants Nos. 11922401, 11734003, 11774028, 11574029), the National Key R\&D Program of China (Grant No. 2016YFA0300600), the Strategic Priority Research Program of Chinese Academy of Sciences (Grant No. XDB30000000).

* ccliu@bit.edu.cn

† ygyao@bit.edu.cn

[1] Y. Cao, V. Fatemi, A. Demir, S. Fang, S. L. Tomarken, J. Y. Luo, J. D. Sanchez-Yamagishi, K. Watanabe, T. Taniguchi, E. Kaxiras, R. C. Ashoori, and P. JarilloHerrero, Nature 556, 80 (2018).

[2] Y. Cao, V. Fatemi, S. Fang, K. Watanabe, T. Taniguchi, E. Kaxiras, and P. Jarillo-Herrero, Nature 556, 43 (2018).

[3] Y. Xie, B. Lian, B. Jäck, X. Liu, C.-L. Chiu, K. Watan- 
abe, T. Taniguchi, B. A. Bernevig, and A. Yazdani, Nature 572, 101 (2019).

[4] A. Kerelsky, L. J. McGilly, D. M. Kennes, L. Xian, M. Yankowitz, S. Chen, K. Watanabe, T. Taniguchi, J. Hone, C. Dean, A. Rubio, and A. N. Pasupathy, Nature 572, 95 (2019).

[5] Y. Jiang, X. Lai, K. Watanabe, T. Taniguchi, K. Haule, J. Mao, and E. Y. Andrei, Nature 573, 91 (2019).

[6] Y. Choi, J. Kemmer, Y. Peng, A. Thomson, H. Arora, R. Polski, Y. Zhang, H. Ren, J. Alicea, G. Refael, F. von Oppen, K. Watanabe, T. Taniguchi, and S. Nadj-Perge, Nature Physics 15, 1174 (2019).

[7] S. L. Tomarken, Y. Cao, A. Demir, K. Watanabe, T. Taniguchi, P. Jarillo-Herrero, and R. C. Ashoori, Phys. Rev. Lett. 123, 046601 (2019).

[8] Y.-Z. You and A. Vishwanath, npj Quantum Materials 4, 16 (2019).

[9] I. Yudhistira, N. Chakraborty, G. Sharma, D. Y. H. Ho, E. Laksono, O. P. Sushkov, G. Vignale, and S. Adam, Phys. Rev. B 99, 140302 (2019).

[10] C.-C. Liu, L.-D. Zhang, W.-Q. Chen, and F. Yang, Phys. Rev. Lett. 121, 217001 (2018).

[11] X. Hu, T. Hyart, D. I. Pikulin, and E. Rossi, Phys. Rev. Lett. 123, 237002 (2019).

[12] H. Isobe, N. F. Q. Yuan, and L. Fu, Phys. Rev. X 8, 041041 (2018).

[13] Y. Da Liao, Z. Y. Meng, and X. Y. Xu, Phys. Rev. Lett. 123, 157601 (2019).

[14] Y.-N. Ren, C. Lu, Y. Zhang, S.-Y. Li, Y.-W. Liu, C. Yan, Z.-H. Guo, C.-C. Liu, F. Yang, and L. He, ACS Nano 14, 13081 (2020).

[15] S.-Y. Li, K.-Q. Liu, L.-J. Yin, W.-X. Wang, W. Yan, X.Q. Yang, J.-K. Yang, H. Liu, H. Jiang, and L. He, Phys. Rev. B 96, 155416 (2017).

[16] Y. Saito, J. Ge, K. Watanabe, T. Taniguchi, and A. F. Young, Nature Physics 16, 926 (2020).

[17] X. Liu, Z. Wang, K. Watanabe, T. Taniguchi, O. Vafek, and J. Li, arXiv preprint arXiv:2003.11072 (2020).

[18] L. Zou, H. C. Po, A. Vishwanath, and T. Senthil, Phys. Rev. B 98, 085435 (2018).

[19] N. F. Q. Yuan and L. Fu, Phys. Rev. B 98, 045103 (2018).

[20] M. Koshino, N. F. Q. Yuan, T. Koretsune, M. Ochi, K. Kuroki, and L. Fu, Phys. Rev. X 8, 031087 (2018).

[21] J. Kang and O. Vafek, Phys. Rev. X 8, 031088 (2018).

[22] H. C. Po, L. Zou, T. Senthil, and A. Vishwanath, Phys. Rev. B 99, 195455 (2019).

[23] S. Carr, S. Fang, H. C. Po, A. Vishwanath, and E. Kaxiras, Phys. Rev. Research 1, 033072 (2019).

[24] J. Ahn, S. Park, and B.-J. Yang, Phys. Rev. X 9, 021013 (2019).

[25] Z. Song, Z. Wang, W. Shi, G. Li, C. Fang, and B. A. Bernevig, Phys. Rev. Lett. 123, 036401 (2019).

[26] J. Liu, J. Liu, and X. Dai, Phys. Rev. B 99, 155415 (2019).

[27] G. Tarnopolsky, A. J. Kruchkov, and A. Vishwanath, Phys. Rev. Lett. 122, 106405 (2019).

[28] H. Guo, X. Zhu, S. Feng, and R. T. Scalettar, Phys. Rev. B 97, 235453 (2018).

[29] B. Roy and V. Juričić, Phys. Rev. B 99, 121407 (2019).

[30] X.-C. Wu, A. Keselman, C.-M. Jian, K. A. Pawlak, and C. Xu, Phys. Rev. B 100, 024421 (2019).

[31] F. Wu, A. H. MacDonald, and I. Martin, Phys. Rev. Lett. 121, 257001 (2018).

[32] J. González and T. Stauber, Phys. Rev. Lett. 122, 026801
(2019).

[33] H. C. Po, L. Zou, A. Vishwanath, and T. Senthil, Phys. Rev. X 8, 031089 (2018).

[34] T. Huang, L. Zhang, and T. Ma, Science Bulletin 64, 310 (2019).

[35] S. Liu, E. Khalaf, J. Y. Lee, and A. Vishwanath, arXiv preprint arXiv:1905.07409 (2019).

[36] C. Lu, Y. Zhang, Y. Zhang, M. Zhang, C.-C. Liu, Z.-C. Gu, W.-Q. Chen, and F. Yang, arXiv preprint arXiv:2003.09513 (2020).

[37] N. Bultinck, E. Khalaf, S. Liu, S. Chatterjee, A. Vishwanath, and M. P. Zaletel, Phys. Rev. X 10, 031034 (2020).

[38] J. Liu and X. Dai, Preprint at https://arxiv. org/abs/1911.03760 (2020).

[39] Y. Zhang, K. Jiang, Z. Wang, and F. Zhang, Phys. Rev. B 102, 035136 (2020).

[40] M. Xie and A. H. MacDonald, Phys. Rev. Lett. 124, 097601 (2020).

[41] C. Shen, Y. Chu, Q. Wu, N. Li, S. Wang, Y. Zhao, J. Tang, J. Liu, J. Tian, K. Watanabe, et al., Nature Physics 16, 520 (2020).

[42] X. Liu, Z. Hao, E. Khalaf, J. Y. Lee, Y. Ronen, H. Yoo, D. H. Najafabadi, K. Watanabe, T. Taniguchi, A. Vishwanath, et al., Nature 583, 221 (2020).

[43] Y. Cao, D. Rodan-Legrain, O. Rubies-Bigorda, J. M. Park, K. Watanabe, T. Taniguchi, and P. Jarillo-Herrero, Nature 583, 215 (2020).

[44] N. R. Chebrolu, B. L. Chittari, and J. Jung, Phys. Rev. B 99, 235417 (2019).

[45] G. W. Burg, J. Zhu, T. Taniguchi, K. Watanabe, A. H. MacDonald, and E. Tutuc, Phys. Rev. Lett. 123, 197702 (2019).

[46] Y. W. Choi and H. J. Choi, Phys. Rev. B 100, 201402 (2019).

[47] M. Koshino, Phys. Rev. B 99, 235406 (2019).

[48] J. Y. Lee, E. Khalaf, S. Liu, X. Liu, Z. Hao, P. Kim, and A. Vishwanath, Nature communications 10, 1 (2019).

[49] Y.-T. Hsu, F. Wu, and S. Das Sarma, Phys. Rev. B 102, $085103(2020)$.

[50] W.-J. Zuo, J.-B. Qiao, D.-L. Ma, L.-J. Yin, G. Sun, J.-Y. Zhang, L.-Y. Guan, and L. He, Phys. Rev. B 97, 035440 (2018).

[51] Y. Shi, S. Xu, M. M. A. Ezzi, N. Balakrishnan, A. GarciaRuiz, B. Tsim, C. Mullan, J. Barrier, N. Xin, B. A. Piot, et al., arXiv preprint arXiv:2004.12414 (2020).

[52] A. Vela, M. V. O. Moutinho, F. J. Culchac, P. Venezuela, and R. B. Capaz, Phys. Rev. B 98, 155135 (2018).

[53] J. Liu, Z. Ma, J. Gao, and X. Dai, Phys. Rev. X 9, 031021 (2019).

[54] X. Li, F. Wu, and A. H. MacDonald, arXiv preprint arXiv:1907.12338 (2019).

[55] Z. Zhu, S. Carr, D. Massatt, M. Luskin, and E. Kaxiras, Phys. Rev. Lett. 125, 116404 (2020).

[56] G. Chen, L. Jiang, S. Wu, B. Lyu, H. Li, B. L. Chittari, K. Watanabe, T. Taniguchi, Z. Shi, J. Jung, Y. Zhang, and F. Wang, Nature Physics 15, 237 (2019).

[57] B. L. Chittari, G. Chen, Y. Zhang, F. Wang, and J. Jung, Phys. Rev. Lett. 122, 016401 (2019).

[58] J. Kang, J. Li, S.-S. Li, J.-B. Xia, and L.-W. Wang, Nano letters 13, 5485 (2013).

[59] Y. Li and M. Koshino, Phys. Rev. B 99, 075438 (2019).

[60] F. Wu, T. Lovorn, E. Tutuc, and A. H. MacDonald, Phys. Rev. Lett. 121, 026402 (2018). 
[61] L. Xian, D. M. Kennes, N. Tancogne-Dejean, M. Altarelli, and A. Rubio, Nano Letters 19, 4934 (2019).

[62] R. Bistritzer and A. H. MacDonald, Proceedings of the National Academy of Sciences 108, 12233 (2011).

[63] E. J. Mele, Phys. Rev. B 84, 235439 (2011).

[64] J. M. B. Lopes dos Santos, N. M. R. Peres, and A. H. Castro Neto, Phys. Rev. Lett. 99, 256802 (2007).

[65] Z. F. Wang, F. Liu, and M. Y. Chou, Nano Letters, Nano Letters 12, 3833 (2012).

[66] E. Suárez Morell, J. D. Correa, P. Vargas, M. Pacheco, and Z. Barticevic, Phys. Rev. B 82, 121407 (2010).

[67] S. Shallcross, S. Sharma, E. Kandelaki, and O. A. Pankratov, Phys. Rev. B 81, 165105 (2010).

[68] A. O. Sboychakov, A. L. Rakhmanov, A. V. Rozhkov, and F. Nori, Phys. Rev. B 92, 075402 (2015).

[69] X. Lin and D. Tománek, Phys. Rev. B 98, 081410 (2018).

[70] J. D. Correa, M. Pacheco, and E. S. Morell, Journal of Materials Science 49, 642 (2014).

[71] L. Xian, D. M. Kennes, N. Tancogne-Dejean, M. Altarelli, and A. Rubio, Nano letters 19, 4934 (2019).

[72] K. Uchida, S. Furuya, J.-I. Iwata, and A. Oshiyama, Phys. Rev. B 90, 155451 (2014).

[73] P. Kang, W.-T. Zhang, V. Michaud-Rioux, X.-H. Kong, C. Hu, G.-H. Yu, and H. Guo, Phys. Rev. B 96, 195406 (2017).

[74] P. Lucignano, D. Alfè, V. Cataudella, D. Ninno, and G. Cantele, Phys. Rev. B 99, 195419 (2019).

[75] S. Latil and L. Henrard, Phys. Rev. Lett. 97, 036803 (2006).

[76] A. H. Castro Neto, F. Guinea, N. M. R. Peres, K. S.
Novoselov, and A. K. Geim, Rev. Mod. Phys. 81, 109 (2009).

[77] H. Min and A. H. MacDonald, Phys. Rev. B 77, 155416 (2008).

[78] N. Marzari and D. Vanderbilt, Phys. Rev. B 56, 12847 (1997).

[79] I. Souza, N. Marzari, and D. Vanderbilt, Phys. Rev. B 65, 035109 (2001).

[80] G. Kresse and J. Hafner, Phys. Rev. B 47, 558 (1993).

[81] P. E. Blöchl, Phys. Rev. B 50, 17953 (1994).

[82] J. P. Perdew, J. A. Chevary, S. H. Vosko, K. A. Jackson, M. R. Pederson, D. J. Singh, and C. Fiolhais, Phys. Rev. B 46, 6671 (1992).

[83] J. P. Perdew, K. Burke, and M. Ernzerhof, Phys. Rev. Lett. 78, 1396 (1997).

[84] H. J. Monkhorst and J. D. Pack, Phys. Rev. B 13, 5188 (1976).

[85] A. A. Mostofi, J. R. Yates, G. Pizzi, Y.-S. Lee, I. Souza, D. Vanderbilt, and N. Marzari, Computer Physics Communications 185, 2309 (2014).

[86] https://github.com/jincao2013/twistwanTB .

[87] D. J. Thouless, M. Kohmoto, M. P. Nightingale, and M. den Nijs, Phys. Rev. Lett. 49, 405 (1982).

[88] Y. Yao, L. Kleinman, A. H. MacDonald, J. Sinova, T. Jungwirth, D.-s. Wang, E. Wang, and Q. Niu, Phys. Rev. Lett. 92, 037204 (2004).

[89] X. Wang, J. R. Yates, I. Souza, and D. Vanderbilt, Phys. Rev. B 74, 195118 (2006).

[90] T. Fukui, Y. Hatsugai, and H. Suzuki, Journal of the Physical Society of Japan 74, 1674 (2005).

\section{Appendix A: Calculation methods}

In this work, the electronic structures for $\mathrm{TMG}_{\mathrm{M}}^{\mathrm{N}}$ are obtained from the effective continuum method[62]. The band structures for the untwisted N-layer and M-layer FLG are adapted from the ab initio results (see detail in Appendix.C). The ab initio calculations for FLG were performed in the VASP package[80] and the electron-ion interaction was described using the projector augmented wave (PAW) method[81]. The exchange-correlation part was described with the generalized gradient approximation (GGA)[82] in the scheme of Perdew-Burke-Ernzerhof (PBE) functional[83]. The plane-wave cutoff energy was set to be $400 \mathrm{eV}$. The Brillouin zone (BZ) was sampled by a $\Gamma$ centered Monkhorst-Pack grid $(12 \times 12 \times 1)$ [84]. The lattice constant for all FLG is set at $2.46 \AA$. The Wannier tight-binding model for FLG was constructed by the WANNIER90 code[85].

\section{Appendix B: Orbital character of few-layer graphene}

In this section, we present the orbitals character for FLG with either chirally and multi-chirally stacking order from ab initio calculations. The band projections around $\mathbf{K}$ valley for chirally stacked FLG are presented in Fig.S.1. The low energy states are well described by the pseudospin doublet with approximately quadratic, cubic and quartic dispersion for $\mathrm{AB}, \mathrm{ABC}$ and $\mathrm{ABCA}$ stacking order respectively. The Fermi surface wrapping effects are automatically taken into account. By projecting onto the $p_{z}$ orbitals at zero mode sites [highlight in red in Fig.S.1(a), (c) and (e)], we found the low energy states, which dominate the physics in the absence of twist angle, are mainly distributed in these zero mode sites. The contributions of the rest $p_{z}$ orbitals are far away from the Fermi energy.

For multi-chirally stacked FLG, we numerically calculated ABA and ABCAC stacked FLG, which can be decomposed into $\{\mathrm{AB}\}\{\mathrm{A}\}$ and $\{\mathrm{ABCA}\}\{\mathrm{C}\}$ respectively. The band structures near $\mathbf{K}$ valley are illustrated in Fig.S.2. The low energy states for ABA stacked FLG are two pseudospin doublets in linear and quadratic dispersion respectively, and the rest bands are pushed into high energy indicating a well preserved chiral decomposition rule despite a generic 

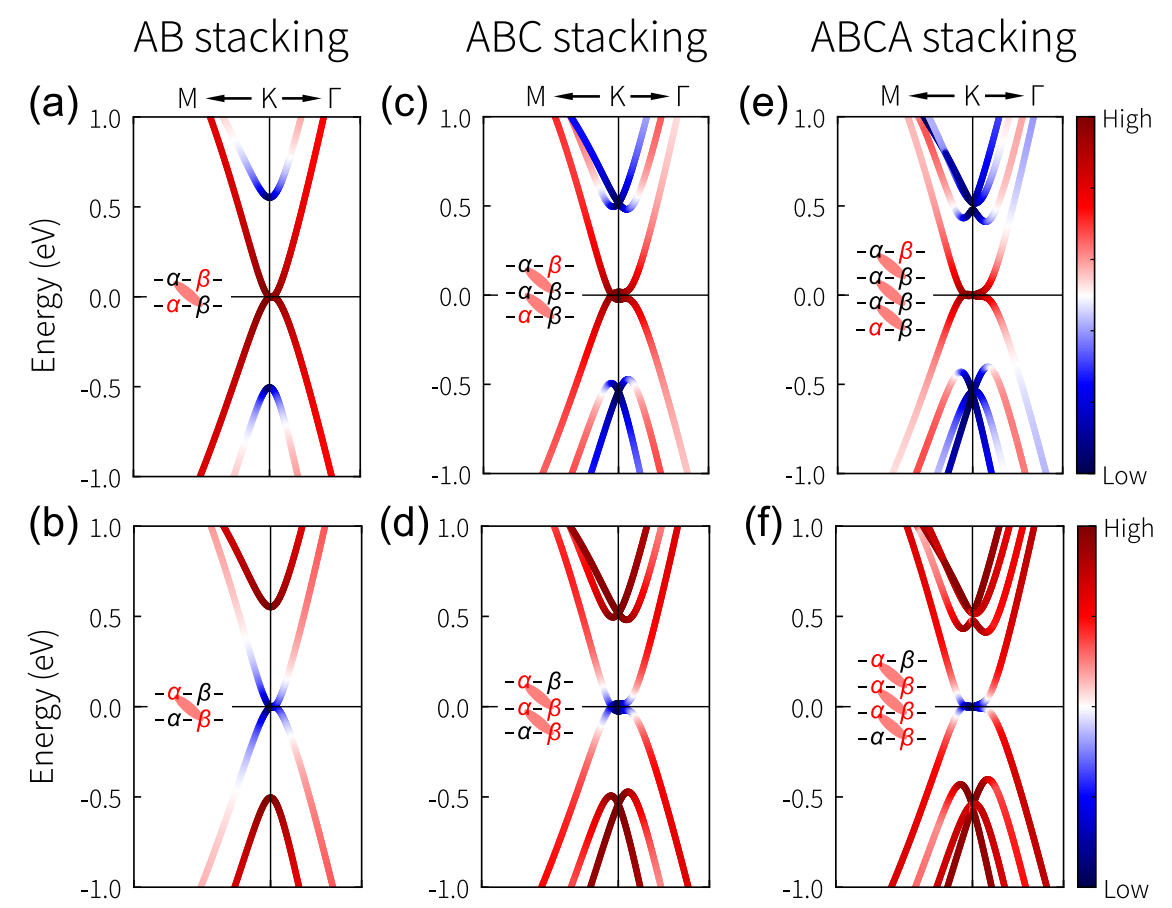

FIG. S.1. Projected band structures near $\mathbf{K}$ valley for chirally stacked few-layer graphene with stacking order AB, ABC and ABCA respectively. The selected $p_{z}$ orbitals are colored in red as shown inside each subfigure. (a),(c) and (e) show the projection on the top and bottom layer, which mainly dominated the low energy states. (b), (d) and (f) show the projection on the rest part, which is almost distributed at high energy states.

hopping parameters are included in our ab initio results. Again, by projecting onto the $p_{z}$ orbitals at zero mode sites [highlight in red in Fig.S.2(a) and (c)], we found they mainly contribute to the two doublets respectively. The rest $p_{z}$ orbitals contribute to the high energy bands. Similar results are obtained for ABCAC stacked FLG as shown in Fig.S.2.

In summary, the chiral decomposition rules are well preserved in the ab initio results. Moreover, the low energy pseudospin doublets in FLG, which play an important role in the presence of twist angle, are mainly contributed from the $p_{z}$ orbitals localized in the zero mode sites. The Fermi surface wrapping effect are automatically taken into account.

\section{Appendix C: The effective continuum model for generic twisted graphene systems}

In this section, we illustrate the combination of ab initio Wannier TB model for FLG and the effective continuum method[62] used in this work to obtain the single particle electronic structures for generic twisted multilayer graphene system (TMG). The geometric structure is illustrated in Fig.1 in the main text. There are N layers graphene on the top and $\mathrm{M}$ layers graphene on the bottom with small twist angles $\pm \theta / 2$ respectively. The emerged moiré pattern is labeled as $\mathbf{L}^{\mathrm{M}}=l_{1} \mathbf{L}_{1}^{\mathrm{M}}+l_{2} \mathbf{L}_{2}^{\mathrm{M}}$. The Bloch states are constructed from the microscopic $p_{z}$ orbitals in each layer of graphene $\left|\tau, d, \mathbf{L}^{\mathrm{M}}+\mathbf{R}+\mathbf{d}\right\rangle$. Here $\tau=\tau_{\alpha}, \tau_{\beta}$ represents graphene sublattice degree of freedom and $d$ is the layer index measured from the bottom to the top (for TDBG, $d=-2,-1,1,2$ from the bottom to the top layer). $\mathbf{R}$ represents the graphene unit cell (in each moiré pattern). $\mathbf{d}=d d_{0} \hat{\mathbf{z}}$ represent the layer stacking distance where $d_{0}$ is the distance between two graphene layers. The Bloch sum functions read as

$$
\left|\tilde{\psi}_{\mathbf{k}_{\xi}^{d}+\mathbf{G}}^{\left(\xi ; \tau_{\alpha}, d\right)}\right\rangle=\sum_{\mathbf{L}^{\mathrm{M}}, \mathbf{R}} e^{i\left(\mathbf{k}_{\xi}^{d}+\mathbf{G}\right) \cdot \mathcal{D}\left[\operatorname{sign}(d) \frac{\theta}{2}\right]\left(\mathbf{L}^{\mathrm{M}}+\mathbf{R}+\tau_{\alpha}\right)}\left|\tau_{\alpha}, d, \mathbf{L}^{\mathrm{M}}+\mathbf{R}+\mathbf{d}\right\rangle
$$

The summations run over all graphene unit cell in Born-von Karman supercell. Here $\xi \equiv \xi_{ \pm}= \pm 1$ represents different graphene valleys. $\mathbf{k}_{\xi}^{d}=\mathbf{k}+\mathbf{K}_{\xi}^{\mathrm{FLG}}-\mathbf{K}_{\xi}^{d}$ is measured from the $\Gamma$ point in the graphene Brillouin zone, and 

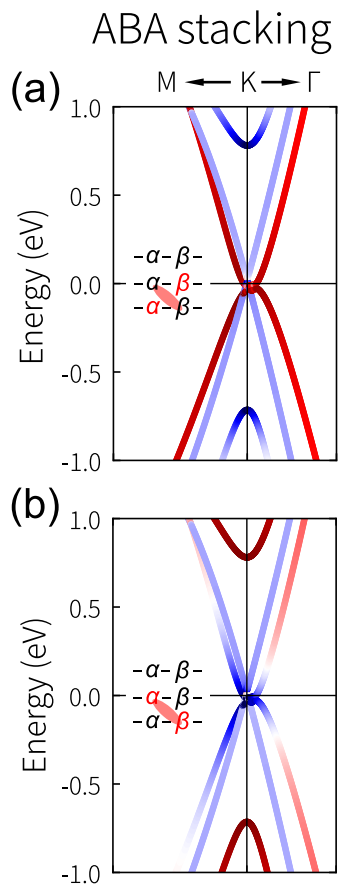

(e) ABCAC stacking

(d)
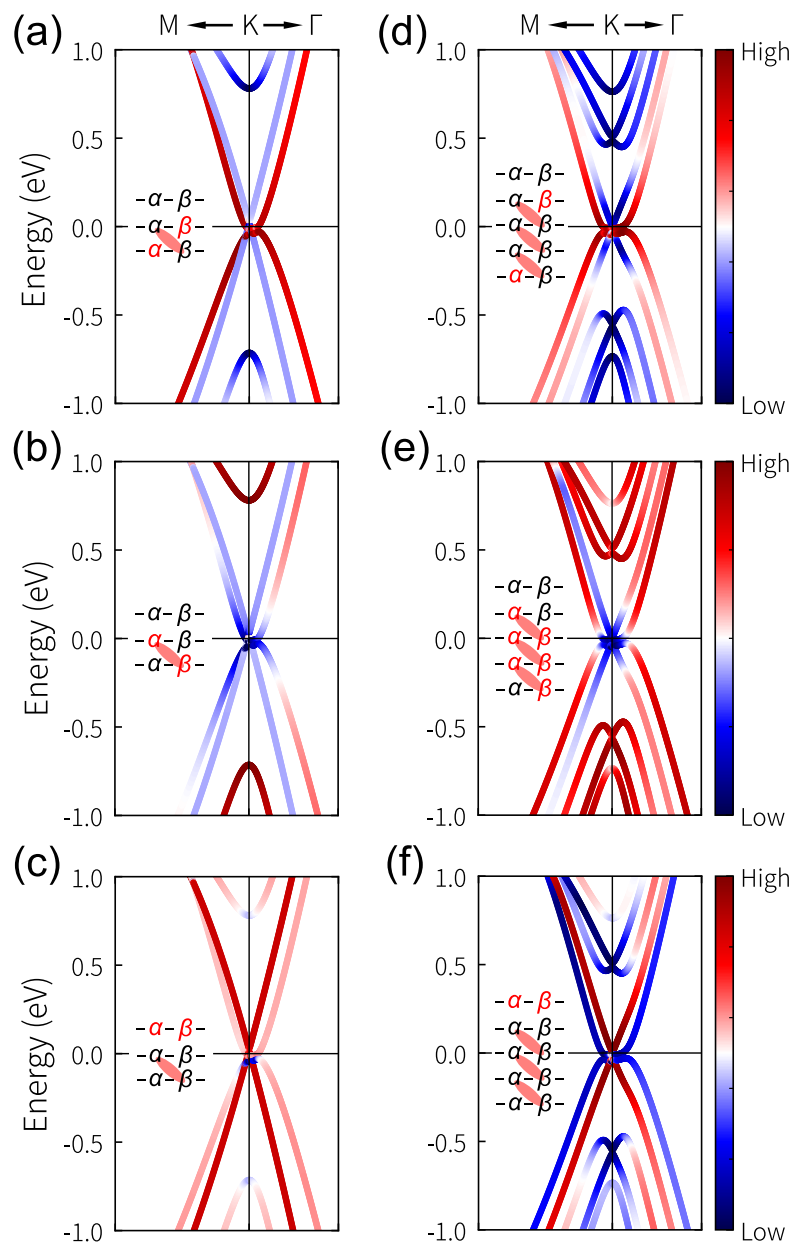

(f)

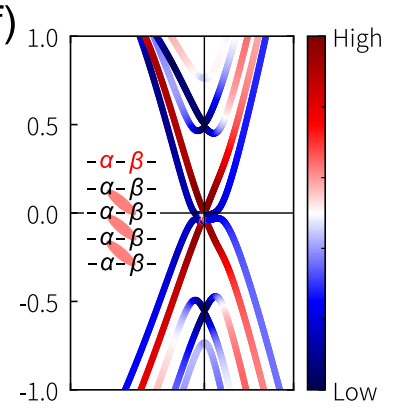

FIG. S.2. Projected band structures near $\mathbf{K}$ valley for multi-chirally stacked few-layer graphene with stacking order ABA and ABCAC respectively. The selected $p_{z}$ orbitals are colored in red as illustrated inside each subfigure.

$\mathbf{k}$ is measured from the $\Gamma$ point in the TMG Brillouin zone. $\mathbf{K}_{\xi}^{\mathrm{FLG}}=\frac{\xi}{3}\left(2 \mathbf{G}_{1}^{\mathrm{FLG}}+\mathbf{G}_{2}^{\mathrm{FLG}}\right)$ is the graphene valley, $\mathbf{K}_{\xi}^{d>0}=\frac{\xi}{3}\left(\mathbf{G}_{1}^{\mathrm{M}}-\mathbf{G}_{2}^{\mathrm{M}}\right)$ and $\mathbf{K}_{\xi}^{d<0}=-\frac{\xi}{3}\left(\mathbf{G}_{1}^{\mathrm{M}}+2 \mathbf{G}_{2}^{\mathrm{M}}\right) \cdot \mathbf{G}=n_{1} \mathbf{G}_{1}^{\mathrm{M}}+n_{2} \mathbf{G}_{2}^{\mathrm{M}}$ is the reciprocal lattice vector for TMG, $\mathcal{D}\left[\operatorname{sign}(d) \frac{\theta}{2}\right]$ indicates a twisted angle $\theta / 2$ for the top part and $-\theta / 2$ for the bottom part. The low energy states can be expended as the Bloch sum functions near the two valleys

$$
\begin{aligned}
\left|\psi_{n \mathbf{k}}\right\rangle & =\sum_{\xi ; \tau, d} \sum_{\mathbf{G}} C_{n \mathbf{k}}^{(\xi ; \tau, d)}(\mathbf{G})\left|\tilde{\psi}_{\mathbf{k}_{\xi}^{d}+\mathbf{G}}^{(\xi ; \tau, d)}\right\rangle \\
& =\sum_{X \mathbf{G}} C_{n \mathbf{k}}^{X}(\mathbf{G})\left|\tilde{\psi}_{\mathbf{k}_{\xi}^{d}+\mathbf{G}}^{X}\right\rangle,
\end{aligned}
$$

where we have rewritten $X=(\xi ; \tau, d)$ for simplicity. $C_{n \mathbf{k}}^{X}(\mathbf{G})$ can be obtained by diagonalizing the effective continuum model

$$
\hat{H}(k)=\hat{H}_{0}(k)+\hat{H}_{\mathrm{T}}+\hat{H}_{\mathrm{BN}}+\hat{H}_{\mathrm{D}}
$$

where $\hat{H}_{0}$ describes the few-layer graphene in the top and bottom part, and $\hat{H}_{\mathrm{T}}$ is the effective twisted interlayer coupling. The $\mathcal{C}_{2 z}$ symmetry can be removed by considering the effect of h-BN substrate $\hat{H}_{\mathrm{BN}}$. And $\hat{H}_{\mathrm{D}}$ describes the 
displacement field to separate the flat bands. In the Bloch sum basis Eq.(C1), the Hamiltonian matrix elements read as

$$
\begin{aligned}
\left\langle\tilde{\psi}_{\mathbf{k}_{\xi}^{d}+\mathbf{G}}^{\left(\xi ; \tau_{\alpha}, d\right)}\left|\hat{H}_{0}\right| \tilde{\psi}_{\mathbf{k}_{\xi^{\prime}}^{d^{\prime}+\mathbf{G}^{\prime}}}^{\left(\xi^{\prime} ; \tau_{\beta}, d^{\prime}\right)}\right\rangle= & \delta_{\xi \xi^{\prime}} \delta_{\operatorname{sign}(d), \operatorname{sign}\left(d^{\prime}\right)} \delta_{\mathbf{G G}^{\prime}} \\
& \times \sum_{\mathbf{R}} e^{i\left(\mathbf{k}_{\xi}^{d}+\mathbf{G}\right) \cdot \mathbf{R}}\left\langle\tau_{\alpha}, d, \mathbf{0}+\mathbf{d}\left|\hat{H}_{0}\right| \tau_{\beta}, d^{\prime}, \mathbf{R}+\mathbf{d}^{\prime}\right\rangle,
\end{aligned}
$$

which are obtained by Fourier transforming the Wannier tight-binding model of FLG. The matrix elements for $\hat{H}_{\mathrm{BN}}$ are

$$
\left\langle\tilde{\psi}_{\mathbf{k}_{\xi}^{d}+\mathbf{G}}^{\left(\xi ; \tau_{\alpha}, d\right)}\left|\hat{H}_{\mathrm{BN}}\right| \tilde{\psi}_{\mathbf{k}_{\xi^{\prime}}^{\prime}+\mathbf{G}^{\prime}}^{\left(\xi^{\prime} ; \tau_{\beta}, d^{\prime}\right)}\right\rangle=\delta_{\xi \xi^{\prime}} \delta_{d d^{\prime}} \delta_{\mathbf{G G}^{\prime}} \sigma_{\alpha \beta}^{z} \Delta_{\mathrm{BN}}^{d}
$$

The matrix elements for $\hat{H}_{\mathrm{D}}$ are

$$
\left\langle\tilde{\psi}_{\mathbf{k}_{\xi}^{d}+\mathbf{G}}^{\left(\xi ; \tau_{\alpha}, d\right)}\left|\hat{H}_{\mathrm{D}}\right| \tilde{\psi}_{\mathbf{k}_{\xi^{\prime}}^{d^{\prime}}+\mathbf{G}^{\prime}}^{\left(\xi^{\prime} ; d^{\prime}, d^{\prime}\right.}\right\rangle=\delta_{\xi \xi^{\prime}} \delta_{d d^{\prime}} \delta_{\mathbf{G G}^{\prime}} \times \sigma_{\alpha \beta}^{0} U_{\mathrm{D}}^{d}
$$

where we define $U_{\mathrm{D}} \equiv U_{\mathrm{D}}^{\max (d)}-U_{\mathrm{D}}^{\min (d)}$. The twisted interlayer coupling read as

$$
\begin{aligned}
&\left\langle\tilde{\psi}_{\mathbf{k}_{\xi}^{d}+\mathbf{G}}^{\left(\xi ; \tau_{\alpha}, d\right)}\left|\hat{H}_{\mathrm{T}}\right| \tilde{\psi}_{\mathbf{k}_{\xi^{\prime}}^{d^{\prime}+\mathbf{G}^{\prime}}}^{\left(\xi^{\prime} ; \tau_{\beta}, d^{\prime}\right)}\right\rangle= \delta_{\xi \xi^{\prime}} \delta_{d= \pm 1, d^{\prime}=\mp 1} \\
& \times\left[T_{1} \delta_{\mathbf{G}, \mathbf{G}^{\prime}}+T_{2} \delta_{\mathbf{G}, \mathbf{G}^{\prime}+\xi \mathbf{G}_{1}^{\mathrm{M}}}+T_{3} \delta_{\left.\mathbf{G}, \mathbf{G}^{\prime}+\xi\left(\mathbf{G}_{1}^{\mathrm{M}}+\mathbf{G}_{2}^{\mathrm{M}}\right)\right]}\right. \\
& T_{1}=\left(\begin{array}{cc}
u & u^{\prime} \\
u^{\prime} & u
\end{array}\right), \quad T_{2}=\left(\begin{array}{cc}
u & u^{\prime} \omega^{-\xi} \\
u^{\prime} \omega^{\xi} & u
\end{array}\right), \quad T_{3}=\left(\begin{array}{cc}
u & u^{\prime} \omega^{\xi} \\
u^{\prime} \omega^{-\xi} & u
\end{array}\right)
\end{aligned}
$$

$\omega=e^{2 \pi i / 3}$. The twisted interlayer coupling parameters $u=0.0797 \mathrm{eV}$ and $u^{\prime}=0.0975 \mathrm{eV}$, which take the relaxation effect into account[20].

\section{Appendix D: Building Wannier tight-binding model}

In this section, we present the details of building the Wannier tight-binding model for TMG following the methodology built-in WANNIER90[78, 79]. As illustrated in the main text, it is possible to choose a group of WFs $\left\{\left|g_{n}\right\rangle\right\}$ in moiré pattern scales to represent the low energy flat bands

$$
\left|g_{n}\right\rangle=\frac{1}{2} \sum_{\xi} \sum_{\tau, d, \mathbf{L}^{\mathrm{M}}, \mathbf{R}} e^{i \mathbf{K}_{\xi}^{\mathrm{FLG}} \cdot \mathbf{r}} f_{n}^{(\xi ; \tau, d)}(\mathbf{r})\left|\tau, d, \mathbf{L}^{\mathrm{M}}+\mathbf{R}+\mathbf{d}\right\rangle,
$$

where the high frequency part is explicitly presented and $f_{n}^{(\xi ; \tau, d)}$ is the smooth envelope function in moiré length scale. With the constraint of real-valued WFs, i.e., $f_{n}^{\left(\xi_{+} ; \tau, d\right)}=f_{n}^{\left(\xi_{-} ; \tau, d\right)} \equiv f_{n}^{(\tau, d)}$, it follows

$$
\left|g_{n}\right\rangle=\sum_{\tau, d, \mathbf{L}^{\mathrm{M}}, \mathbf{R}} \cos \left(\mathbf{K}_{\xi}^{\mathrm{FLG}} \cdot \mathbf{r}\right) f_{n}^{(\tau, d)}(\mathbf{r})\left|\tau, d, \mathbf{L}^{\mathrm{M}}+\mathbf{R}+\mathbf{d}\right\rangle,
$$

which indicates an equal mixture of two valleys. The initial guess for the Bloch sum functions are obtained by projecting the initial WFs $g_{n}$ onto the Bloch states of TMG

$$
\left|\tilde{\phi}_{n \mathbf{k}}^{(0)}\right\rangle=\sum_{m}\left|\psi_{m \mathbf{k}}\right\rangle\left\langle\psi_{m \mathbf{k}} \mid g_{n}\right\rangle
$$




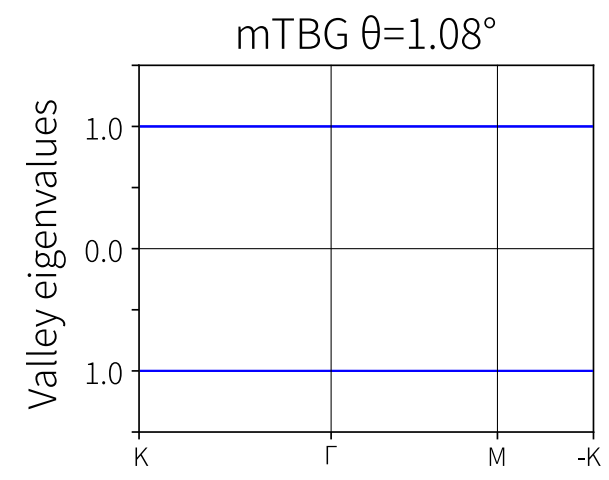

FIG. S.3. Valley eigenvalues interpolated from real space valley operator of TBG with twist angle $\theta=1.08^{\circ}$.

The orbital projection matrix $\left\langle\psi_{m \mathbf{k}} \mid g_{n}\right\rangle$ can be calculated as

$$
\begin{gathered}
A_{m n}(\mathbf{k})=\left\langle\psi_{m \mathbf{k}} \mid g_{n}\right\rangle=\sum_{X \mathbf{G}} C_{m \mathbf{k}}^{X}(\mathbf{G})^{*}\left\langle\tilde{\psi}_{\mathbf{k}_{\xi}^{d}+\mathbf{G}}^{X} \mid g_{n}\right\rangle \\
\left\langle\tilde{\psi}_{\mathbf{k}_{\xi}^{d}+\mathbf{G}}^{X} \mid g_{n}\right\rangle=\frac{1}{2 \pi} \int d \mathbf{r} e^{-i\left(\mathbf{k}_{\xi}^{d}+\mathbf{G}\right) \cdot \mathcal{D}\left[\operatorname{sign}(d) \frac{\theta}{2}\right] \mathbf{r}} \cos \left(\mathbf{K}_{\xi}^{\mathrm{FLG}} \cdot \mathbf{r}\right) f_{n}^{(\tau, d)}(\mathbf{r}) .
\end{gathered}
$$

The last equation is the inner product of the initial Wannier orbital and the Bloch sum function. We then preform the singular value decomposition (SVD) to orthogonalize the initial Bloch sum

$$
\left|\tilde{\phi}_{n \mathbf{k}}^{(1)}\right\rangle=\sum_{m}\left|\psi_{m \mathbf{k}}\right\rangle\left(A_{\mathbf{k}} S_{\mathbf{k}}^{-1 / 2}\right)_{m n}
$$

where

$$
\begin{aligned}
A_{\mathbf{k}} & =U_{\mathbf{k}} \Sigma_{\mathbf{k}} V_{\mathbf{k}}^{\dagger} \\
S_{\mathbf{k}}^{-1 / 2} & =V_{\mathbf{k}} \frac{1}{\sqrt{\Sigma_{\mathbf{k}}^{\dagger} \Sigma_{\mathbf{k}}}} V_{\mathbf{k}}^{\dagger} .
\end{aligned}
$$

To well describe the subspace of flat bands, we project $\tilde{\phi}_{n \mathbf{k}}^{(1)}$ onto the subspace spanned by flat bands

$$
\begin{aligned}
& \left|\tilde{\phi}_{n \mathbf{k}}^{(2)}\right\rangle=\mathcal{P}_{\mathbf{k}}^{(f . b .)}\left|\tilde{\phi}_{n \mathbf{k}}^{(1)}\right\rangle, \\
& \mathcal{P}_{\mathbf{k}}^{(f . b .)}=\sum_{n}\left|\psi_{n \mathbf{k}}^{(f . b .)}\right\rangle\left\langle\psi_{n \mathbf{k}}^{(f . b .)}\right| .
\end{aligned}
$$

This procedure was first introduced by D. Vanderbilt et al. to well describe certain range of the Bloch bands[79]. Once we get the proper Bloch wave function, the Wannier tight-binding model can be interpolated by

$$
H_{m n}(\mathbf{R})=\frac{1}{N_{\mathbf{k}}} \sum_{\mathbf{k}} e^{-i \mathbf{k} \cdot \mathbf{R}}\left\langle\tilde{\phi}_{m \mathbf{k}}^{(2)}\left|E_{\mathbf{k}} I\right| \tilde{\phi}_{n \mathbf{k}}^{(2)}\right\rangle
$$

where $E_{\mathbf{k}}$ is eigenvalue obtained from the effective continuum model.

To clarify the valley degree of freedom in the present framework, we first define the valley operator in continuum model 
(a)

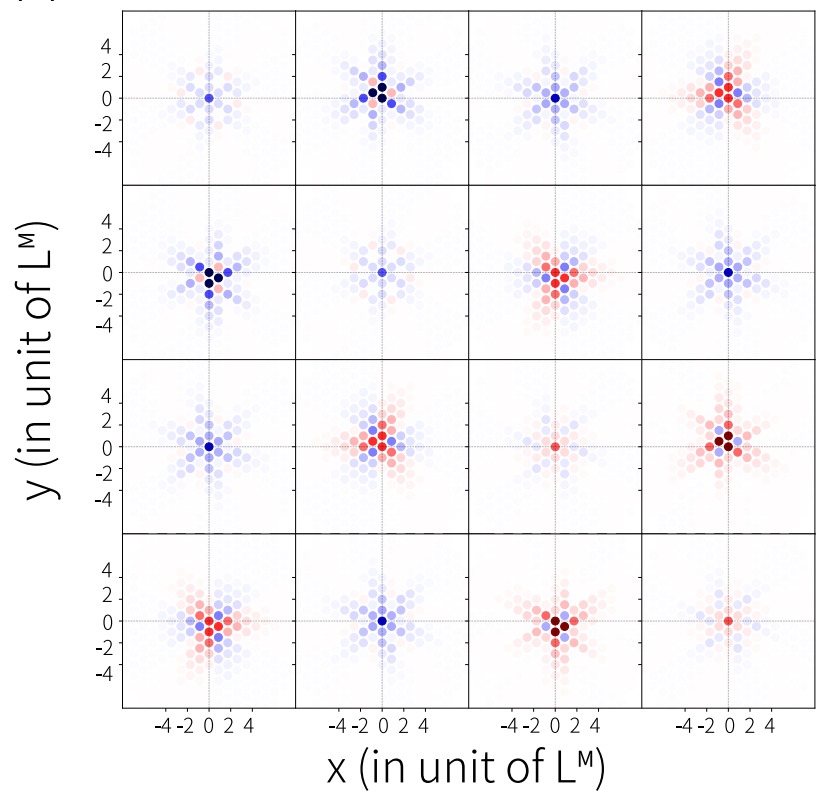

(b)

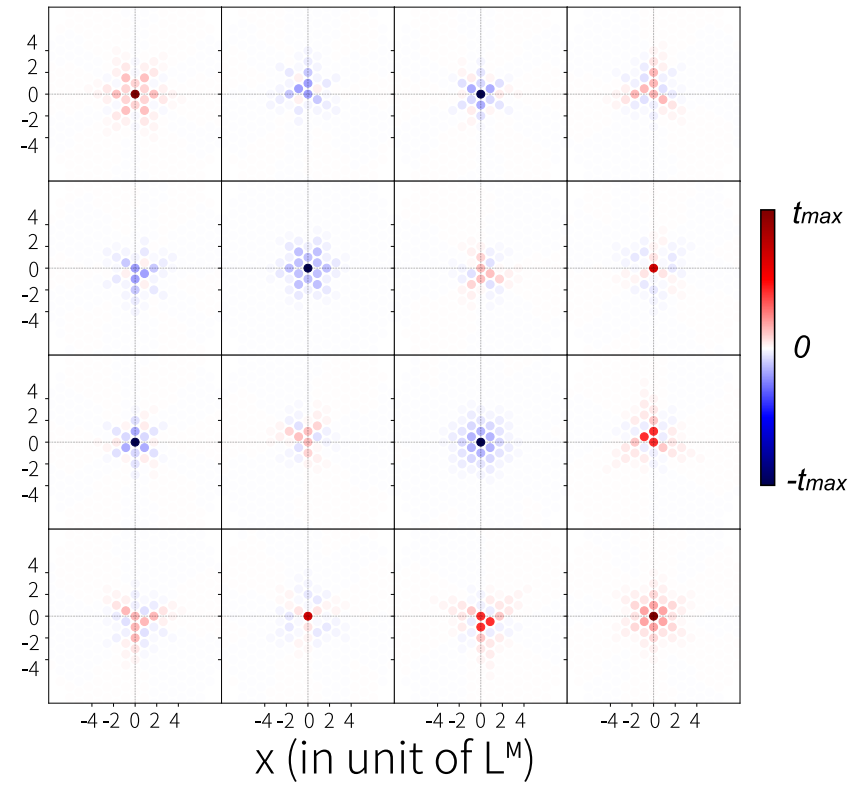

FIG. S.4. Real space hopping parameters between WFs for (a) the magic angle TBG, and (b) the magic angle TBG with the effect of h-BN substrate. The positive values are shown in red whereas the negative ones are shown in blue.

$$
\mathcal{V}_{\mathbf{k}}=\sum_{\xi ; \tau, d} \sum_{\mathbf{G}} \xi\left|\tilde{\psi}_{\mathbf{k}_{\xi}^{d}+\mathbf{G}}^{(\xi ; \tau, d)}\right\rangle\left\langle\tilde{\psi}_{\mathbf{k}_{\xi}^{d}+\mathbf{G}}^{(\xi ;, d)}\right|
$$

Then, the valley operator for the Wannier TB model can be interpolated following the exactly same procedure for the Hamiltonian

$$
\mathcal{V}_{m n}(\mathbf{R})=\frac{1}{N_{\mathbf{k}}} \sum_{\mathbf{k}} e^{-i \mathbf{k} \cdot \mathbf{R}}\left\langle\tilde{\phi}_{m \mathbf{k}}^{(2)}\left|\mathcal{V}_{\mathbf{k}}\right| \tilde{\phi}_{n \mathbf{k}}^{(2)}\right\rangle .
$$

From the real space Hamiltonian $H(\mathbf{R})$ and the valley operator $\mathcal{V}(\mathbf{R})$ one can get the Hamiltonian and the valley operator at certain $\mathbf{k}$ point. The Hamiltonian can be explicitly classified into two decoupled blocks labeled with valley eigenvalues \pm 1 respectively. The valley labeled Bloch states can be obtained by simultaneously diagonalizing the two operators.

A uniform $18 \times 18$ mesh for the Brillouin zone were used for interpolate all of the Wannier tight-binding model in this work. The hopping parameters are real numbers since the WFs are real-valued functions. The $\mathcal{C}_{3 z}$ symmetry is enforced to build WFs and so do the hopping parameters. The Hamiltonian matrix elements are shown in Fig.S.4, Fig.S.5 and Fig.S.6. The resulting Wannier tight-binding models are well documented and available in GitHub[86]. It serves as a start point for further study of many-body effects in TMG systems. The eigenvalues for the valley operator of TBG with twist angle $\theta=1.08^{\circ}$ are shown in Fig.S.3. It is stabilized in \pm 1 . Similar results are obtained for other presented systems.

\section{Appendix E: Chern number for continuum model and Wannier TB model}

In this section, we compare the Berry curvature distributions calculated from the continuum model and Wannier TB model. The Berry curvature for the $\mathrm{n} t h$ band can be calculated from[87, 88]

$$
\Omega_{n}^{z}(\mathbf{k})=-2 \operatorname{Im} \sum_{m \neq n} \frac{v_{m n}^{x}(\mathbf{k}) v_{n m}^{y}(\mathbf{k})}{\left(\omega_{m \mathbf{k}}-\omega_{n \mathbf{k}}\right)^{2}}
$$


(a)

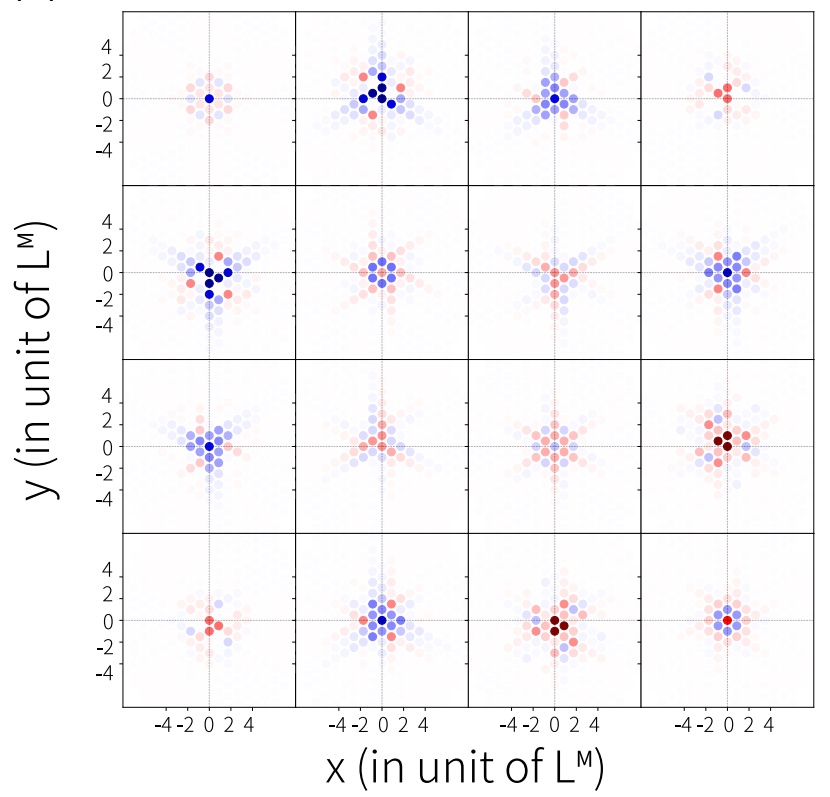

(b) $\operatorname{mTDBG} \theta=1.248^{\circ} \cup_{\mathrm{D}}=10 \mathrm{meV}$

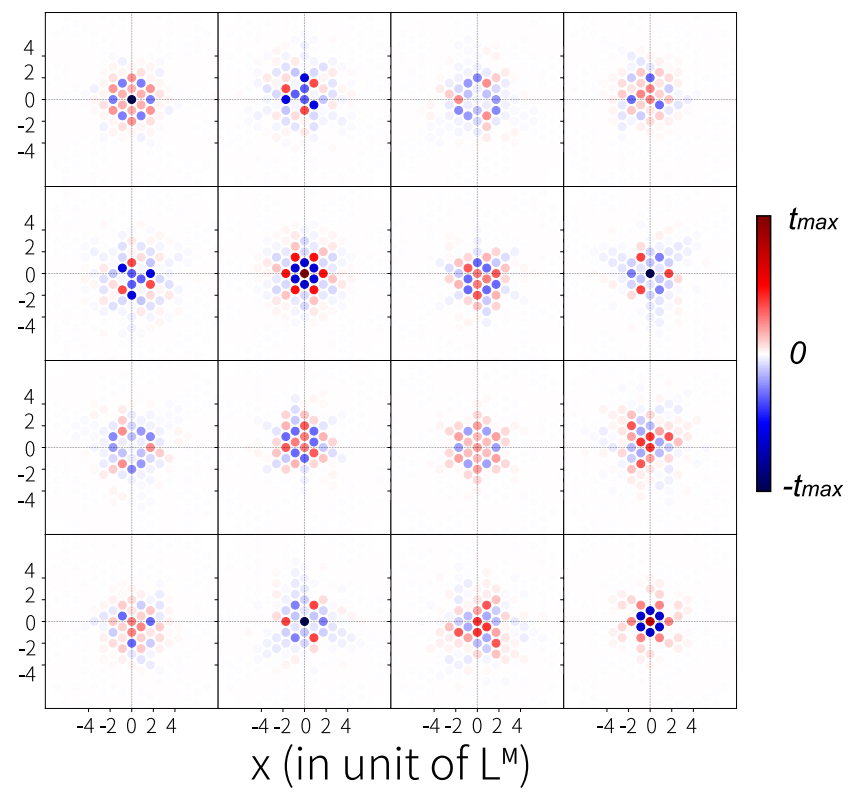

FIG. S.5. Real space hopping parameters between WFs for (a) the TDBG, and (b) the magic angle TDBG with the displacement field turned on $\left(U_{\mathrm{D}}=10 \mathrm{meV}\right)$. The positive values are shown in red whereas the negative ones are shown in blue.

(a) $\operatorname{mTTG} \theta=1.248^{\circ}$ with $\mathrm{hBN} U_{\mathrm{D}}=80 \mathrm{meV}$

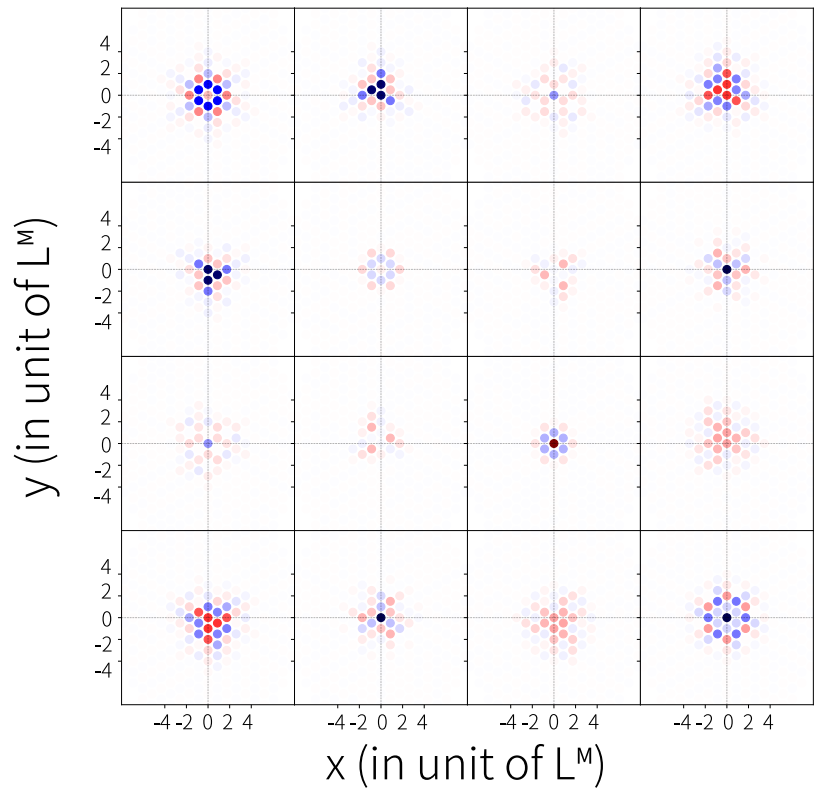

(b)

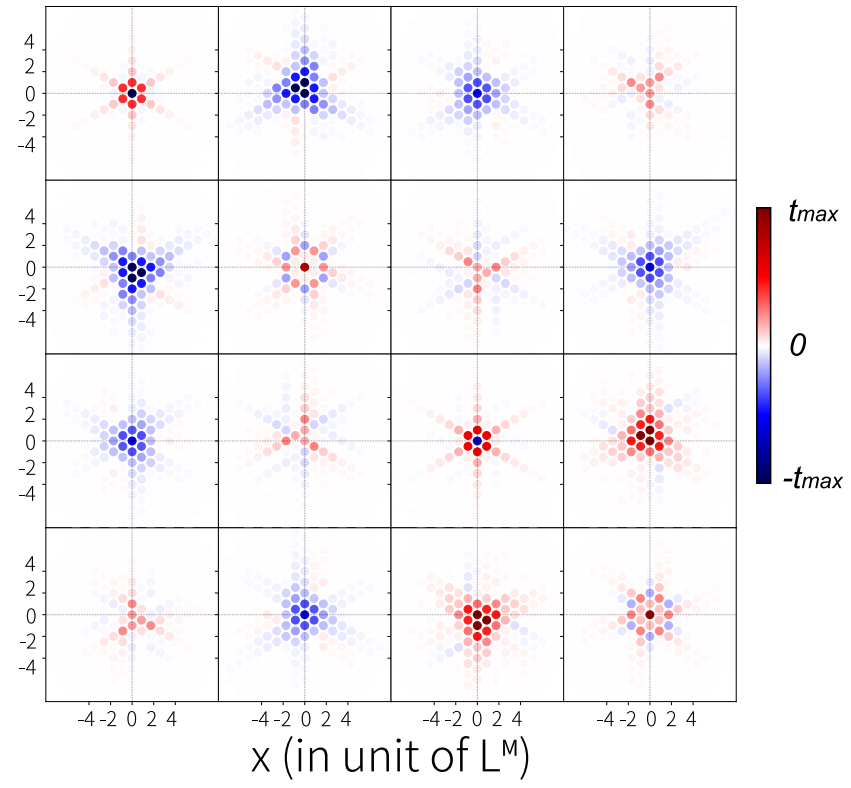

FIG. S.6. Real space hopping parameters between WFs for (a) the magic angle TTG $\left(\mathrm{TMG}_{\{\mathrm{AB}\}}^{\{\mathrm{AB}}\right)$ with h-BN substrate and the displacement field turned on $\left(U_{\mathrm{D}}=80 \mathrm{meV}, \Delta_{\mathrm{BN}}^{d=-1}=80 \mathrm{meV}\right.$ and $\left.\Delta_{\mathrm{BN}}^{d=1}=\Delta_{\mathrm{BN}}^{d=2}=-20 \mathrm{meV}\right)$, and (b) the TMG $\{\mathrm{ABCA}\}$. The positive values are shown in red whereas the negative ones are shown in blue. 


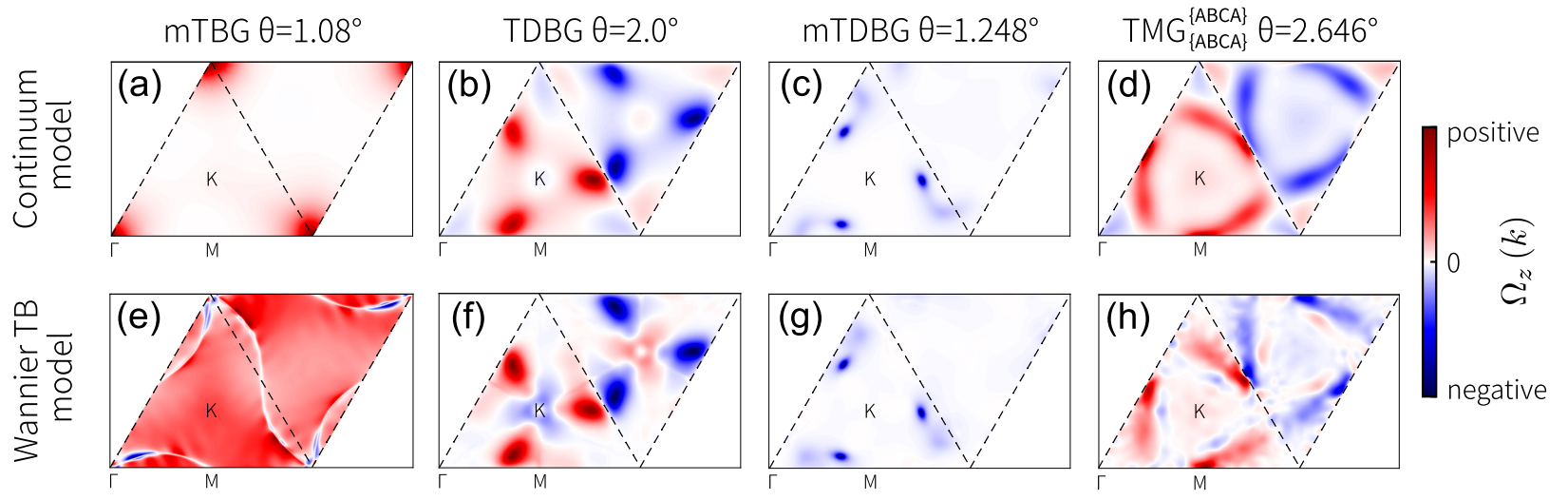

FIG. S.7. Berry curvature distributions for the valence flat band (i.e., the lower flat band) from graphene valley $\mathbf{K}_{\xi}^{\mathrm{FLG}}$ obtained from the continuum model (a)-(d) and Wannier TB model (e)-(h). The high symmetric points in the moiré BZ are presented. The parameters of h-BN substrate $\Delta_{\mathrm{BN}}^{d}$ and displacement field $U_{\mathrm{D}}$ are given in the main text. The interpolated Wannier TB model can well describe the Berry curvature distributions, so does the Chern number.

where $\varepsilon_{n \mathbf{k}}=\hbar \omega_{n \mathbf{k}}$ and $\boldsymbol{v}(\mathbf{k})$ is the velocity operator matrix. And the Chern number is followed by integration over the two-dimensional BZ

$$
\mathcal{C}_{n}=\frac{1}{2 \pi} \int d^{2} k \Omega_{n}^{z}(\mathbf{k})
$$

For Wannier TB model, the velocity operator $\boldsymbol{v}(\mathbf{k})$ can be calculated by Wannier interpolation method[89]. It should be notice that due to the extended shape of WFs for TMG systems, one should include more matrix elements $\langle n \mathbf{0}|\hat{\mathbf{r}}| m \mathbf{R}\rangle[$ see Eq.(39) in Ref.[89]] to precisely describe the velocity operator for Wannier TB model. It can be obtained by numerically integrating the WFs in real space. We found however, by including only the Wannier center $\langle n \mathbf{0}|\hat{\mathbf{r}}| n \mathbf{0}\rangle$, one can get reasonable results in Berry curvature calculations. For the continuum model

$$
\begin{aligned}
v_{m n}^{a}(\mathbf{k}) & =\sum_{X X^{\prime}} \sum_{\mathbf{G} \mathbf{G}^{\prime}} C_{m \mathbf{k}}^{X}(\mathbf{G})^{*}\left\langle\tilde{\psi}_{\mathbf{k}_{\xi}^{d}+\mathbf{G}}^{X}\left|i \hbar^{-1}\left[\hat{H}, \hat{r}^{a}\right]\right| \tilde{\psi}_{\mathbf{k}_{\xi^{\prime}}^{d^{\prime}}+\mathbf{G}^{\prime}}^{X^{\prime}}\right\rangle C_{n \mathbf{k}}^{X^{\prime}}\left(\mathbf{G}^{\prime}\right) \\
& =\sum_{X X^{\prime}} \sum_{\mathbf{G G}^{\prime}} C_{m \mathbf{k}}^{X}(\mathbf{G})^{*}\left\langle\tilde{u}_{\mathbf{k}_{\xi}^{d}+\mathbf{G}}^{X}\left|\frac{\partial \hat{H}(\mathbf{k})}{\hbar \partial k_{a}}\right| \tilde{u}_{\mathbf{k}_{\xi^{\prime}}^{d^{\prime}}}^{X^{\prime}+\mathbf{G}^{\prime}}\right\rangle C_{n \mathbf{k}}^{X^{\prime}}\left(\mathbf{G}^{\prime}\right),
\end{aligned}
$$

where

$$
\begin{aligned}
\left\langle\tilde{u}_{\mathbf{k}_{\xi}^{d}+\mathbf{G}}^{X}\left|\frac{\partial \hat{H}(\mathbf{k})}{\hbar \partial k_{a}}\right| \tilde{u}_{\mathbf{k}_{\xi^{\prime}}^{d^{\prime}}+\mathbf{G}^{\prime}}^{X^{\prime}}\right\rangle= & \delta_{\xi \xi^{\prime}} \delta_{\operatorname{sign}(d), \operatorname{sign}\left(d^{\prime}\right)} \delta_{\mathbf{G G}^{\prime}} \\
& \times i \sum_{\mathbf{R}}\left(R^{a}+\tau_{\beta}^{a}-\tau_{\alpha}^{a}\right) e^{i\left(\mathbf{k}_{\xi}^{d}+\mathbf{G}\right) \cdot\left(\mathbf{R}+\tau_{\beta}-\tau_{\alpha}\right)}\left\langle\tau_{\alpha}, d, \mathbf{0}+\mathbf{d}\left|\hat{H}_{0}\right| \tau_{\beta}, d^{\prime}, \mathbf{R}+\mathbf{d}^{\prime}\right\rangle .
\end{aligned}
$$

The Berry curvature distributions of TBG, TDBG and TMG for both the continuum model and Wannier TB model are shown in Fig.S.7. It can be well described by the interpolated Wannier TB model. The numerical integration of the Berry curvature are presented in TABLE.III giving the Chern number for the flat band. For TMG system with vanishing valley Chern number (the two flat bands together for single valley have zero Chern number), our model gives the correct topological classification comparing with the original continuum model. For TMG system with non-vanishing valley Chern number, for instance the mTTG in our presented results, despite the well fitted band structure, our current model fails to describe the topological feature for mTTG. 
TABLE. III. Chern number for twisted multilayer graphenes. It is obtained by integrating the Berry curvature over the BZ [see Eq.(E2)]. The same results are obtained from Wilson loop method and efficient lattice method[90]. Here $\xi_{+}$and $\xi_{-}$label $\left(\mathbf{K}, \mathbf{K}^{\prime}\right)$ valley, and $\mathrm{VB}, \mathrm{CB}$ indicate the lower and higher flat bands respectively.

\begin{tabular}{lcccccc}
\hline \hline & Flat band index & mTBG $\left(1.08^{\circ}\right)$ & $\mathrm{TDBG}\left(2.0^{\circ}\right)$ & $\mathrm{mTDBG}\left(1.248^{\circ}\right)$ & $\mathrm{mTTG}^{\mathrm{T}}\left(1.248^{\circ}\right)$ & $\mathrm{TMG}_{\{\mathrm{ABCA}\}}^{\{\mathrm{ABCA}\}}\left(2.646^{\circ}\right)$ \\
\hline Continuum model & $\left(\xi_{-}, \mathrm{VB}\right)$ & 0.975 & 0.021 & -3.061 & -0.014 & 0.188 \\
& $\left(\xi_{-}, \mathrm{CB}\right)$ & -0.996 & -0.031 & 3.027 & 0.954 & -0.182 \\
& $\left(\xi_{+}, \mathrm{VB}\right)$ & -0.976 & -0.021 & 3.061 & 0.014 & -0.183 \\
& $\left(\xi_{+}, \mathrm{CB}\right)$ & 0.996 & 0.031 & -3.027 & -0.954 & 0.176 \\
Wannier TB model & $\left(\xi_{-}, \mathrm{VB}\right)$ & 0.907 & 0.011 & -2.873 & -0.053 & 0.055 \\
& $\left(\xi_{-}, \mathrm{CB}\right)$ & -0.981 & 0.004 & 2.929 & -0.053 & -0.055 \\
& $\left(\xi_{+}, \mathrm{VB}\right)$ & -0.907 & -0.011 & 2.873 & -0.127 & -0.008 \\
\hline \hline
\end{tabular}

